\title{
MODELS AND METHODS FOR THREE EXTERNAL BALLISTICS INVERSE PROBLEMS
}

\author{
N.K. Arutyunova, A.M. Dulliev, V.I. Zabotin \\ Kazan National Research Technical University named after A.N. Tupolev - KAI, Kazan, \\ Russian Federation \\ E-mail: NKArutyunova@kai.ru, AMDulliev@kai.ru, VIZabotin@kai.ru
}

\begin{abstract}
We consider three problems of selecting optimal gun barrel direction (or those of selecting optimal semi-axis position) when firing an unguided artillery projectile on the assumption that the gun barrel semi-axis can move in a connected nonconvex cone having a non-smooth lateral surface and modelling visibility zone restrictions. In the first problem, the target is in the true horizon plane of the gun, the second and the third problems deal with some region of $3 \mathrm{D}$ space. A distinctive feature of the models is that the objective functions are $\varepsilon$-Lipschitz ones. We have constructed a unified numerical method to solve these problems based on the algorithm of projecting a point onto $\varepsilon$-Lipschitz level function set. A computer programme has been based on it. A series of numerical experiments on each problem has been carried out.

Keywords: mathematical modelling; inverse problem of external ballistics; optimization; E-Lipschitz; projection onto nonconvex set; approximate solution.
\end{abstract}

\section{Introduction}

Let us consider a problem of firing an unguided projectile. It is required to minimize the distance from the projectile drop point to the target. This problem is a problem of the external ballistic theory. It has been studied fairly well (see [1]) if the following conditions hold: air resistance is not taken into account, movement of the gun barrel is restricted by the true horizon plane of the gun, and the Earth surface is spherical.

However, in reality, a gun barrel direction can be arbitrarily selected, as a rule, only within some connected nonconvex cone that has non-smooth lateral surface and arises in conditions narrowing the selecting gun barrel direction due to some obstacles.

To restrictions on the selecting gun barrel direction, it is often necessary to add conditions such that the target can lie outside of the true horizon plane of the gun or, possibly, on the surface defining the relief of a landscape. In the latter case, the problem of selecting optimal gun barrel direction becomes much more complicated. The foremost reason for this difficulty is that the minimal Euclidean distance from the target to the projectile drop point does not always correspond to the optimal shot or even close to the optimal one. For example, this lack of correspondence can be observed in case the target and the projectile drop point are separated from each other by some substantial obstacle.

A mathematical model can be described as follows. Suppose dist(, ) is the Euclidean distance, $O$ is the point in which located the gun, $l$ is a ray with origin $O, N$ is the 
projectile drop point, $N=N(l), M$ is the target, $K$ is a cone with vertex $O, D^{\prime}$ is a set in space $\mathbb{R}^{3}$, and $M \in D^{\prime}$. It is required

$$
\begin{gathered}
\min _{l} \operatorname{dist}(N(l), M), \\
\text { subject to } l \in K, N(l) \in D^{\prime} .
\end{gathered}
$$

Naturally, there may be other possible mathematical models with other objective functions. In particular, below we consider a problem in which the objective function is the distance between the projectile trajectory and the point $M$.

We stress that, in this paper, air resistance is not taken into account. At the same time, first, our models that are described and investigated below can be applied for firing an unguided heavy projectile. Secondly, the solutions obtained in the models can be served as the base for further finding for a more precise solution and can be refined by appropriate methods.

Below, we formulate and investigate three problems for which a unified algorithm for their solving is constructed. Also, we present numerical results for several test examples; these results were obtained with a computer programme implementing the algorithm.

\section{Formulation of the Problems}

Throughout, the gun is modelled by a point $T$, the direction of the gun barrel is modelled by a ray starting from this point, the oblateness of the Earth is not taken into account.

Let us choose a Cartesian coordinate system $O x y z$ with the origin $O$ coinciding with $T$. Suppose the plane $O x y$ is the horizontal plane at $O$, the axis $O z$ is directed vertically up, and the gravitational acceleration $\boldsymbol{g}$, vertically down. The directions of the axes $O x$ and $O y$ will be given below. Throughout, we use the following notation: $\|\cdot\|_{p}$ is the $p$-norm on $\mathbb{R}^{n}, n \geqslant 1$ (the subscript $p$ will be omitted for $p=2$ ); int and fr are the operators of taking interior and boundary in $\mathbb{R}^{n}$, respectively; $\operatorname{Pr}_{i} A$ is the projection of a set $A$ on the $i$-th axis (here, the axes $x, y$, and $z$ are denoted by 1,2 , and 3 , respectively); given $X \subset \mathbb{R}^{n}$ and $Y \subset \mathbb{R}$, we denote by $\operatorname{Lip}(X ; Y)$ the set of all Lipschitz continuous on $X$ functions $f: X \rightarrow Y$ with Lipschitz constant $\operatorname{lip}(f)$.

We will assume that the direction (i.e. ray position) of the gun barrel can be freely selected within a closed cone $K$ with the apex $O$ and the cone $K$ contains no vertical rays starting from $O$. Neglecting the length of the gun barrel, suppose the initial projectile velocity vector $\boldsymbol{v}$ has the initial point at $O$ and has a constant length $v_{0}$. In other words, $\boldsymbol{v} \in S \cap K$, where $S=\left\{\boldsymbol{v} \mid\|\boldsymbol{v}\|=v_{0}\right\} \subset \mathbb{R}^{3}$ is the sphere of radius $v_{0}$ centered at $O$. By $v$ denote the fraction $v_{0} / \sqrt{\|\boldsymbol{g}\|}$.

Throughout, we assume that the selecting direction of the gun barrel is equivalent to the selecting the vector $\boldsymbol{v}=\left(v_{x}, v_{y}, v_{z}\right)$ with a given fixed $v_{0}$. This vector will be described by means of a spherical coordinate system in the form

$$
\begin{gathered}
v_{x}=v_{0} \cos \psi \cos \varphi, \quad v_{y}=v_{0} \cos \psi \sin \varphi, \quad v_{z}=v_{0} \sin \psi, \\
(\varphi, \psi) \in E \subset \Theta:=\{(\varphi, \psi) \mid \varphi \in[0,2 \pi), \psi \in(-\pi / 2, \pi / 2)\},
\end{gathered}
$$

where the closed set $E$ corresponds to the set $S \cap K$, which is given by coordinates $(\varphi, \psi)$.

We assume that the air resistance is negligible. It follows that the trajectory travelled by a projectile is a parabolic trajectory on the gun plane. 
In this paper we will consider three problems of selecting optimal gun barrel direction when firing an unguided projectile to the given target. We will suppose that the target $M$ is the point with $y$-coordinate equal to zero and $M$ belongs to the convex set bounded by the paraboloid reached by the projectile (see [1]), and this paraboloid is obtained by the union of all the projectile trajectories on the condition of the constraints absence on gun barrel directions. (The case, where the target can lie outside of the paraboloid, and the problem of the joint selecting optimal gun barrel direction $(\varphi, \psi)$ and magnitude $v_{0}$ of the initial projectile velocity are not considered in this paper.)

Among the points lying on the projectile trajectories, we will consider only the points with $x$-coordinate such that $x \geqslant \kappa$, where $\kappa=$ const and $\kappa>0$. In practical terms, the constant $\kappa$, for example, may correspond to the blast radius of a projectile.

Other constraints on $x, y$, and $z$-coordinates are different and depend on the problems that will be considered below.

Problem I. Let the point $M$ be the target, lie on the plane $x O y$, and have coordinates $(a, 0), a>\kappa$. Suppose the projectile drop point $N$ also lies on the plane $x O y$ and has coordinates $(x, y)$. For all the trajectories, there are no barriers determined by $\boldsymbol{v} \in S \cap K$. Out of all those trajectories, it is required to choose one for which the Euclidean distance between the points $M$ and $N$ is minimal.

Following [1], the distance $r$ from $O$ to $N(x, y)$ is calculated by

$$
r=\sqrt{x^{2}+y^{2}}=v^{2} \sin 2 \psi .
$$

Using (1) and (2), we see that the coordinates $x$ and $y$ are determined from

$$
x=r \cos \varphi=v^{2} \sin 2 \psi \cos \varphi, \quad y=r \sin \varphi=v^{2} \sin 2 \psi \sin \varphi .
$$

Determined by $(3)$ the map $(\varphi, \psi) \mapsto(x, y)$ from $\Theta$ to $\mathbb{R}^{2}$ is denoted by $h_{\mathrm{I}}$.

Since $v$ is fixed, we obtain the upper bound $\rho_{\mathrm{I}}=v^{2}$ for the distance travelled by a projectile. Let us introduce notation

$$
W_{\mathrm{I}}=\left\{(x, y) \mid \sqrt{x^{2}+y^{2}} \leqslant \rho_{\mathrm{I}}, x \geqslant \kappa\right\} .
$$

Obviously, the feasible set of the projectile drop points is given by the set $h_{\mathrm{I}}(E)$.

Thus, the gun barrel direction determined by pair $(\varphi, \psi)$ and the corresponding optimal projectile trajectory can be found by solving the problem

$$
\begin{gathered}
\|M-N\|^{2} \rightarrow \min , \\
\text { s.t. } N \in h_{\mathrm{I}}(E) \cap W_{\mathrm{I}} .
\end{gathered}
$$

In this paper the set $E$ will be described by means of functional inequalities.

Let $\left[\theta_{1}, \theta_{2}\right] \subset[0,2 \pi)$ and the functions $g_{1}(\varphi)$ and $g_{2}(\varphi)$ satisfy the condition

$$
-\pi / 2 \leqslant g_{1}(\varphi) \leqslant g_{2}(\varphi) \leqslant \pi / 2, \varphi \in\left[\theta_{1}, \theta_{2}\right]
$$

We put

$$
E=\left\{(\varphi, \psi) \in \Theta \mid \varphi \in\left[\theta_{1}, \theta_{2}\right], g_{1}(\varphi) \leqslant \psi \leqslant g_{2}(\varphi)\right\}
$$

or, in other words,

$$
E=\{(\varphi, \psi) \in \Theta \mid g(\varphi, \psi) \leqslant 0\}
$$


where

$$
g(\varphi, \psi):=\max \left\{\theta_{1}-\varphi, \varphi-\theta_{2}, g_{1}(\varphi)-\psi, \psi-g_{2}(\varphi)\right\}
$$

Note that Problem I admits a generalization to the case when the target is the set $\mathcal{M}=\left\{M_{1}, M_{2}, \ldots, M_{n}\right\}$ of the points $M_{i}=\left(a_{i}, b_{i}\right), i=1, \ldots, n, n>1$, on the plane $x O y$. Indeed, the Chebyshev center of $\mathcal{M}$ can be choosen as the target at a single shot, for example.

Problem II. Let the point $M(a, 0, c) \in \mathbb{R}^{3}$ be the target. Suppose the target and all the possible projectile drop points lie not below the plane $z=z_{\min }\left(z_{\min }<0\right)$. As in Problem I, assume that $a>\kappa$. We will distinguish two subproblems, which depend on constraints imposed on the point $M$. These subproblems will be named Problem II.a and Problem II.b, respectively.

II.a. Let the point $M$ be an arbitrary point (i.e. $M$ can be in the air) and the projectile trajectory selection is limited only the set E. Among all admissible trajectories, it is required to choose the one for which the distance from it to the target $M$ is minimal in comparison with the distance from any remaining admissible trajectory to the target $M$.

II.b. Let the point $M$ belong to the boundary fr $D$ of a set $D$ determined by inequality $H(x, y, z) \leqslant 0$, where $H$ is a continuous function over $\mathbb{R}^{3}$ and $H(0,0,0) \geqslant 0$. This function defines the relief of a landscape. (It means that the target can be located on a surface of ground/water or some fixed ground object, and the gun, on a surface of ground/water or in the air). We assume that the point $N(x, y, z)$ belongs to fr $D$, the section of the trajectory from $O$ to $N$ has no common points with int $D$, and $z \leqslant z_{\min }$ implies $H(x, y, z) \leqslant 0$. Among all admissible trajectories, it is required to choose the one for which the segment $M N$ does not intersect int $D$ and has minimal length or it is required to be certain that there is no such one. This requirement means that the projectile drop point must be within the line-of-sight of the target and be located maximally close to the target.

Note that the target can lie above $(c>0)$ or below $(c<0)$ relative to the plane $x O y$ and the optimal value of angle $\psi$ can be less than zero when $c<0$.

Before giving analytical formulations of Problem II.a and Problem II.b, let us make some important remarks.

Unlike Problem I, for Problems II.a and II.b, it is either impossible or very difficult to specify a single-valued map from $\Theta$ to $\mathbb{R}^{3}$ that analogous to $h_{\mathrm{I}}$. A reason for this difficulty is that the target and the coverage zone in Problems II.a and II.b are located in $\mathbb{R}^{3}$. Speaking in more detail, in Problem II.a, to each point $(\varphi, \psi) \in E$ there corresponds not one point but a subset of the set of projectile trajectory points; in Problems II.b an analytical determination of coordinates of the point $N(x, y, z)$ for given $(\varphi, \psi) \in E$ may be realized in only special cases that is defined by the set $D$.

It is convenient to consider a single-valued map instead of a set-valued map from $\Theta$ to $\mathbb{R}^{3}$. This map, denoted below by $h_{\mathrm{II}}: \Theta \times \mathbb{R}^{+} \rightarrow \mathbb{R}^{3}$, takes each $(\varphi, \psi, r) \in \Theta \times \mathbb{R}^{+}$to the point $(x, y, z) \in \mathbb{R}^{3}$ assuming that $(x, y, z)$ lies on the projectile trajectory determined by $(\varphi, \psi)$ and the distance from $O$ to the projection of the point $(x, y, z)$ on the plane $x O y$ is equal to $r$.

According to the theory of external ballistic, the map $h_{\mathrm{II}}$ is determined by

$$
h_{\mathrm{II}}(\varphi, \psi, r)=\left\{(x, y, z) \mid x=r \cos \varphi, y=r \sin \varphi, z=r \tan \psi-\left(1+\tan ^{2} \psi\right)\left(2 v^{2}\right)^{-1} r^{2}\right\} .
$$


Despite single-valuedness of the maps $h_{\mathrm{I}}$ and $h_{\mathrm{II}}$, their inverse maps $h_{\mathrm{I}}^{-1}$ and $h_{\mathrm{II}}^{-1}$ are multivalued: the drop point can be reached by selecting one of the two appropriate values of the angle $\psi \in[-\pi / 2, \pi / 2]$ for the direction of the gun barrel. At the same time, the value of the angle $\varphi$ is uniquely determined. In constructing the functional constraints for Problem I and II.a,b, the maps $h_{\mathrm{I}}^{-1}$ and $h_{\mathrm{II}}^{-1}$ play an important role. A reason, on which this constructing is possible, is that the maps $h_{\mathrm{I}}^{-1}$ and $h_{\mathrm{II}}^{-1}$ can be parameterized by single-valued maps. Indeed, it follows from (3), (7) that there exist the single-valued maps $h_{\mathrm{I} j}^{-1}, h_{\mathrm{II} j}^{-1}, j \in\{1,2\}$ for which

$$
\begin{gathered}
h_{k}^{-1}=h_{k 1}^{-1} \cup h_{k 2}^{-1}, \operatorname{dom}\left(h_{k j}^{-1}\right)=\operatorname{dom}\left(h_{k}^{-1}\right), k \in\{\mathrm{I}, \mathrm{II}\}, j \in\{1,2\} ; \\
\operatorname{Pr}_{2} \operatorname{Im}\left(h_{\mathrm{I} 1}^{-1}\right)=(0, \pi / 4], \operatorname{Pr}_{2} \operatorname{Im}\left(h_{\mathrm{I} 2}^{-1}\right)=[\pi / 4, \pi / 2), \\
\operatorname{Pr}_{2} \operatorname{Im}\left(h_{\mathrm{II} 1}^{-1}\right)=(-\pi / 2, \pi / 2), \operatorname{Pr}_{2} \operatorname{Im}\left(h_{\mathrm{II} 2}^{-1}\right)=(0, \pi / 2) .
\end{gathered}
$$

The explicit expressions for $h_{k j}^{-1}$ will be given below.

Since all the projectile drop points lie not below the plane $z=z_{\min }$ and using (7), the value $r$ can be bounded from above by

$$
\rho_{\mathrm{II}}=v \sqrt{v^{2}-2 z_{\min }} .
$$

Taking into account this bound and the envelope of the family of the possible projectile trajectories, we define the set

$$
W_{\mathrm{II}}=\left\{(x, y, z) \mid \sqrt{x^{2}+y^{2}} \leqslant \rho_{\mathrm{II}}, x \geqslant \kappa, z_{\min } \leqslant z \leqslant \frac{1}{2}\left(v^{2}-\frac{x^{2}+y^{2}}{v^{2}}\right)\right\},
$$

which is the set of all the points reached by a projectile.

In view of the above remarks, we can now give an analytical formulation of Problem II.a in the form

$$
\begin{gathered}
\|M-N\|^{2} \rightarrow \min , \\
\text { s.t. } N \in h_{\mathrm{II}}(E) \cap W_{\mathrm{II}} .
\end{gathered}
$$

For Problem II.b we have such an analytical formulation

$$
\begin{gathered}
\|M-N\|^{2} \rightarrow \min , \\
\text { s.t. } N \in h_{\mathrm{II}}(E) \cap W_{\mathrm{II}} \cap \text { fr } D,\{\lambda N+(1-\lambda) M \mid \lambda \in[0,1]\} \cap \operatorname{int} D=\varnothing, \\
\exists j \in\{1,2\}: h_{\mathrm{II}}\left(\operatorname{Pr}_{1} h_{\mathrm{II} j}^{-1}(N), \operatorname{Pr}_{2} h_{\mathrm{II} j}^{-1}(N),\left[0, \operatorname{Pr}_{3} h_{\mathrm{II} j}^{-1}(N)\right]\right) \cap \operatorname{int} D=\varnothing .
\end{gathered}
$$

Obviously, the last two equations in (11) mean that the section of the trajectory from $O$ to $N$ and the segment $M N$ does not intersect int $D$, respectively.

By $N_{*}$ denote a point that belongs to the solution set of problem (11).

Unfortunately, problem (11) admits the existence of common points, in addition to $N_{*}$, between the segment $M N$ or the section of the trajectory from $O$ to $N_{*}$ and fr $D$. Of course, adding conditions to the constrains of problem (11), we can eliminate this situation. However, as it is easy to show, a formalization of these conditions does not allow us correctly use any optimization method because the feasible set is not closed, moreover, an attempt to take its closure again leads to problem (11). To solve this problem we propose the following procedure. It is necessary to solve problem (11) and if its solution $N_{*}$ does not satisfy the condition given at the beginning of the paragraph, we have to find another solution of problem (11) in the union of sufficiently small closed "ring like" neighborhoods of the points $\left(\operatorname{Pr}_{1} h_{\mathrm{II} 1}^{-1}\left(N_{*}\right), \operatorname{Pr}_{2} h_{\mathrm{II} 1}^{-1}\left(N_{*}\right)\right)$ and $\left(\operatorname{Pr}_{1} h_{\mathrm{II} 2}^{-1}\left(N_{*}\right), \operatorname{Pr}_{2} h_{\mathrm{II} 2}^{-1}\left(N_{*}\right)\right)$, respectively. 


\section{Minimizing the Objective Functions}

Problems (5), (10), and (11) are complex in general case. Despite the fact that the objective functions for these problems are smooth at every point $(x, y, z)$ in their domains, the functions describing the feasible set can be non-differentiable. In particular, this applies to the functions $g_{1}$ and $g_{2}$. Similarly, we can assert that these statements hold for problems (5) and (10) with respect to the variables $\varphi, \psi$ and the variables $\varphi, \psi, r$, respectively. Moreover, problem (11) with respect to the variables $\varphi, \psi, r$ may have both objective function and feasible set functions that are non-differentiable.

Surely, in each problem (5), (10), and (11), we could try smoothing all its functions and then solve the corresponding smoothed problem by invoking some optimization method, for example, linearization method or sequential quadratic programming method. At the same time, a complicated form of the feasible set functions, especially in Problems II.a, II.b, (see below), indicates that using smoothing procedures is not useful, because the ones do not give substantial simplification of the original problem. Moreover, after this smoothing, the solution of any smoothed problem may be outside the visibility zone $E$ for the gun barrel or may be far from the optimal solution.

It is obvious that we can minimize the objective function in problem (5) either with respect to the variables $(\varphi, \psi)$, or with respect to the variables $(x, y)$. Similarly, this holds for problem (10) with respect to the variables $(\varphi, \psi, r)$ and $(x, y, z)$, respectively. In any case, the found optimal solution allows us to find right away the required optimal gun barrel direction, which is determined by $(\varphi, \psi)$, and, for problem (10), to find, in addition, a point closest to the target and lying on the optimal trajectory.

As above, in Problem II.b, we should not rely on an analytical determination of coordinates of the point $N(x, y, z)$ by using the variables $\varphi, \psi$. Therefore, it is reasonable to solve this problem with respect to the variables $x, y, z$. Moreover, we give an argument, which confirms that problems (5) and (10) should be also solved with respect to the variables $x, y$, and $z$.

Thanks to smoothness of the objective functions for problems (5) and (10) with respect to the variables $\varphi, \psi, r$, we can solve these problems using a first-order optimization algorithm. However if the functions $g_{1}$ or $g_{2}$ are non-differentiable or the set $E$ is nonconvex, then the use of this algorithm is difficult, because the conditions for its convergence may be not satisfied or because solving the auxiliary problems, for example, as finding a projection onto $E$, is also difficult.

In this paper we will suppose that the functions $g_{1}$ and $g_{2}$ are only Lipschitz continuous on some solid rectangle contained in $\Theta$. This condition is completely natural, since it can be satisfied, if the visibility zone $E$ has been piecewise linearly approximated on the basis of the photography results.

Following the conditions for the functions $g_{1}$ and $g_{2}$, and following the above remarks about the variables in problems (5), (10), and (11), we will solve all problems with respect to $x, y, z$.

In addition, since the maps $h_{\mathrm{I}}^{-1}$ and $h_{\mathrm{II}}^{-1}$ are multivalued, it follows that the feasible sets in each problems should be presented as the union of two subsets that correspond to $h_{k 1}^{-1}$ and $h_{k 2}^{-1}, k \in\{\mathrm{I}, \mathrm{II}\}$ (see (8)). This implies that instead of one problem either (5), or (10), or (11), we have two corresponding subproblems, which may be solved independently of each other. 
Clearly, each problem (5), (10), or (11) (and, therefore, its subproblems) is a problem of finding a projection the point $M$ onto the feasible set. We claim that the feasible set for each problem can be defined by the corresponding functional inequality in the form

$$
F_{i j}(x, y, z) \leqslant 0, i \in\{\text { I, II.a, II.b }\}, j \in\{1,2\},
$$

where $j$ is the subproblem number and the left-hand side of inequality satisfies so-called the $\varepsilon$-Lipschitz condition. This condition will allow us to use the methods proposed in [2, 3].

For problems $(5)$ and (10), the functions $F_{\text {I } j}$ and $F_{\text {II.a } j}$ can be defined by

$$
\begin{gathered}
F_{\mathrm{I} j}(N):=\max \left\{\omega_{\mathrm{I} j 1} g\left(\operatorname{Pr}_{1} h_{\mathrm{I} j}^{-1}(N), \operatorname{Pr}_{2} h_{\mathrm{I} j}^{-1}(N)\right), \omega_{\mathrm{I} j 2}\left(\kappa-\operatorname{Pr}_{1} N\right)\right\}, \\
F_{\text {II. } j}(N):=\max \left\{\omega_{\text {II.a } j 1} g\left(\operatorname{Pr}_{1} h_{\mathrm{II} j}^{-1}(N), \operatorname{Pr}_{2} h_{\mathrm{II} j}^{-1}(N)\right),\right. \\
\left.\omega_{\text {II.a } j 2}\left(\kappa-\operatorname{Pr}_{1} N\right), \omega_{\mathrm{II} . \mathrm{a} j 3}\left(z_{\text {min }}-\operatorname{Pr}_{3} N\right)\right\} .
\end{gathered}
$$

Here and in what follows, by $\omega_{i j l}$ we denote the weighting coefficients whose values depend on the properties of the functions defining $F_{i j}$. Later on, before presenting the algorithm for solving Problems I, II.a, II.b, we will propose a technique for choosing $\omega_{i j l}$.

Since the constraints for problem (11) can be formalized by

$$
\left\{\begin{array}{l}
g\left(\operatorname{Pr}_{1} h_{\mathrm{II} j}^{-1}(N), \operatorname{Pr}_{2} h_{\mathrm{II} j}^{-1}(N)\right) \leqslant 0, H(N)=0, \operatorname{Pr}_{1} N \geqslant \kappa, \min _{\lambda \in[0,1]}\{H(\lambda N+(1-\lambda) M)\} \geqslant 0, \\
\min _{\mu \in[0,1]}\left\{H\left(h_{\mathrm{II}}\left(\operatorname{Pr}_{1} h_{\mathrm{II} j}^{-1}(N), \operatorname{Pr}_{2} h_{\mathrm{II} j}^{-1}(N), \mu \operatorname{Pr}_{3} h_{\mathrm{II} j}^{-1}(N)\right)\right)\right\} \geqslant 0, z_{\min }-\operatorname{Pr}_{3} N \leqslant 0,
\end{array}\right.
$$

we can define the function $F_{\text {II.b } j}$ by the formula

$$
\begin{aligned}
F_{\text {II.b } j}(N):=\max \{ & \omega_{\text {II.b } j 1} g\left(\operatorname{Pr}_{1} h_{\mathrm{II} j}^{-1}(N), \operatorname{Pr}_{2} h_{\mathrm{II} j}^{-1}(N)\right), \omega_{\mathrm{II} . \mathrm{b} j 2}\left(\kappa-\operatorname{Pr}_{1} N\right), \\
& \omega_{\text {II.b } j 3}|H(N)|,-\omega_{\text {II.b } j 4} \min _{\lambda \in[0,1]}\{H(\lambda N+(1-\lambda) M)\}, \\
& \left.-\omega_{\text {II.b } j 5} \min _{\mu \in[0,1]}\left\{H\left(h_{\mathrm{II}}\left(\operatorname{Pr}_{1} h_{\mathrm{II} j}^{-1}(N), \operatorname{Pr}_{2} h_{\mathrm{II} j}^{-1}(N), \mu \operatorname{Pr}_{3} h_{\mathrm{II} j}^{-1}(N)\right)\right)\right\}\right\} .
\end{aligned}
$$

The upper bounds for the variables $x, y$ and $z$ in the definition of the sets $W_{i}$ (see (4), (9)) are implicitly contained in (12) - (15), since they follow from projectile motion equations and from the condition $N \in h .(E)$. But the lower bounds for the variables $x$ and $z$ are explicitly contained in $(12)-(15)$, since they were introduced additionally, based on practical considerations.

Thus, each Problem $i, i \in\{$ I, II.a, II.b $\}$, for each $j \in\{1,2\}$ has the form

$$
\begin{gathered}
\|M-N\|^{2} \rightarrow \min , \\
\text { s.t. } F_{i j}(N) \leqslant 0 .
\end{gathered}
$$

Obviously, we should not rely on an analytical methods for solving any problem of the form in (16). Among numerical methods, the choice is small due to complicated properties of the functions $F_{i j}$.

At the same time, the functions $F_{i j}, i \in\{$ I, II.a, II.b $\}, j \in\{1,2\}$ satisfy the so-called the $\varepsilon$-Lipschitz condition (see [4]) in the corresponding set $W_{i}$. Now we give the corresponding definition. 
Let $f$ be a function from a subset $A$ contained in a normed space $X$ to a normed space $Y$. The function $f$ is called $\varepsilon$-Lipschitz continuous on $A$, if for any number $0<\varepsilon<\varepsilon_{0}$ there exists some number $L(\varepsilon)>0$ such that for all $x, y \in A$, the following inequality holds

$$
\|f(x)-f(y)\| \leqslant L(\varepsilon)\|x-y\|+\varepsilon .
$$

By $\varepsilon$-Lip $(A ; Y)$ denote the set of $\varepsilon$-Lipschitz continuous on $A$ functions; for a fixed function $f$ and fixed $\varepsilon$, by $\operatorname{lip}(f ; \varepsilon)$ denote the smallest function (i.e., the infimum) of all $L(\varepsilon)$ satisfying $(17)$. (The properties for $\operatorname{lip}(f ; \varepsilon)$ can be found in [2], where it is denoted by $l(\varepsilon)))$.

To present the assertions that each function $F_{i j}, i \in\{$ I, II.a,II.b $\}, j \in\{1,2\}$ is $\varepsilon$ Lipschitz continuous on a certain set in $\mathbb{R}^{n}$ ( $n$ equal to 2 or 3 ), we shall give the explicit formulas for $\operatorname{Pr}_{1} h_{k j}^{-1}(N), \operatorname{Pr}_{2} h_{k j}^{-1}(N), W_{k}, k \in\{\mathrm{I}, \mathrm{II}\}, j \in\{1,2\}$.

Using (3) and (7), we get

$$
\begin{gathered}
\varphi(N):=\operatorname{Pr}_{1} h_{k j}^{-1}(N)=\arctan (y / x), k \in\{\mathrm{I}, \mathrm{II}\}, j \in\{1,2\} ; \\
\psi_{\mathrm{I} j}(N):=\operatorname{Pr}_{2} h_{\mathrm{I} j}^{-1}(N)=\frac{\pi}{4}+(-1)^{j}\left(\frac{\pi}{4}-\frac{1}{2} \arcsin \frac{\sqrt{x^{2}+y^{2}}}{v^{2}}\right), j \in\{1,2\} ; \\
\psi_{\mathrm{II} j}(N):=\operatorname{Pr}_{2} h_{\mathrm{II} j}^{-1}(N)= \\
=\arctan \left(\left(x^{2}+y^{2}\right)^{-1 / 2}\left(v^{2}+(-1)^{j} \sqrt{v^{4}-\left(x^{2}+y^{2}+2 v^{2} z\right)}\right)\right), j \in\{1,2\} .
\end{gathered}
$$

Note there exist some values of the variables $x, y$, and $z$ such that the expression under the last square root in (18) can be negative. But this fact indicates that, for the given magnitude $v_{0}$ of the initial projectile velocity, the coordinates of the projectile drop point can not be equal to those $x, y$, and $z$ for any values of $\psi$.

The $\varepsilon$-Lipschitz continuity of the functions $F_{i j}, i \in\{$ I, II.a,II.b $\}, j \in\{1,2\}$, is formulated below by Propositions 1-3. We also give several lemmas in which some functions that are used in the definitions of the functions $F_{i j}$ are $\varepsilon$-Lipschitz continuous or Lipschitz continuous. For the subsequent estimates of $\operatorname{lip}(\cdot)$ and $\operatorname{lip}(\cdot ; \varepsilon)$, we use the norm $\|\cdot\|_{1}$; we also assume that $g_{1}, g_{2} \in \operatorname{Lip}\left(\left[\theta_{1}, \theta_{2}\right] ; \mathbb{R}\right), H \in \operatorname{Lip}\left(\mathbb{R}^{3} ; \mathbb{R}\right), 2 \varepsilon \in(0, \pi / 2-1)$.

Lemma 1. For the function $g(\varphi, \psi)$ defined by (6), we have $g \in \operatorname{Lip}(\Theta ; \mathbb{R})$ and

$$
\operatorname{lip}(g) \leqslant \max \left\{\operatorname{lip}\left(g_{1}\right), \operatorname{lip}\left(g_{2}\right), 1\right\} .
$$

The proofs of this lemma and the following ones can be found in [6].

Lemma 2. For each $k \in\{\mathrm{I}, \mathrm{II}\}$, it is true that $\varphi(x, y) \in \operatorname{Lip}\left(W_{k} ;[0,2 \pi)\right)$ and

$$
\operatorname{lip}(\varphi) \leqslant \sqrt{2} \kappa^{-1}
$$

Lemma 3. For each $j \in\{1,2\}$, it is true that $\psi_{\mathrm{I} j}(x, y) \in \varepsilon-\operatorname{Lip}\left(W_{\mathrm{I}} ;(0, \pi / 2)\right)$ and

$$
\operatorname{lip}\left(\psi_{\mathrm{I} j} ; \varepsilon\right) \leqslant\left(\sqrt{2} v^{2} \sqrt{1-\tau^{2}(2 \varepsilon)}\right)^{-1}
$$

where $\tau(2 \varepsilon)$ is the unique root of the equation $(\pi / 2-2 \varepsilon-\arcsin \tau) \sqrt{1-\tau^{2}}=1-\tau$ in the interval $[0,1)$. 
Proposition 1. For each $j \in\{1,2\}$, it is true that $F_{\mathrm{I} j}(N) \in \varepsilon-\operatorname{Lip}\left(W_{\mathrm{I}} ; \mathbb{R}\right)$ and

$$
\operatorname{lip}\left(F_{\mathrm{I} j} ; \varepsilon\right) \leqslant \max \left\{\omega_{\mathrm{I} j 1} \operatorname{lip}(g)\left(\operatorname{lip}(\varphi)+\operatorname{lip}\left(\psi_{\mathrm{I} j} ; \varepsilon\left(\omega_{\mathrm{I} j 1} \operatorname{lip}(g)\right)^{-1}\right)\right), \omega_{\mathrm{I} j 2}\right\},
$$

where $\operatorname{lip}(g)$, lip $(\varphi)$, and $\operatorname{lip}\left(\psi_{\mathrm{I} j} ; \varepsilon\left(\omega_{\mathrm{I} j 1} \operatorname{lip}(g)\right)^{-1}\right)$ determined by (19), (20), and (21), respectively.

Lemma 4. For each $j \in\{1,2\}$, it is true that $\psi_{\mathrm{II} j}(N) \in \varepsilon-\operatorname{Lip}\left(W_{\mathrm{II}} ;(-\pi / 2, \pi / 2)\right)$ and

$$
\operatorname{lip}\left(\psi_{\mathrm{II} j} ; \varepsilon\right) \leqslant\left(\rho_{\mathrm{II}}(2 \varepsilon)^{-1}+\beta \sqrt{2}\right) \kappa^{-2},
$$

where $\beta=v^{2}+\sqrt{v^{4}-\kappa^{2}-2 v^{2} z_{\min }}$.

Proposition 2. For each $j \in\{1,2\}$, it is true that $F_{\text {II. } a j}(N) \in \varepsilon-\operatorname{Lip}\left(W_{\text {II }} ; \mathbb{R}\right)$ and

$$
\operatorname{lip}\left(F_{\text {II. } a j} ; \varepsilon\right) \leqslant \max \left\{\omega_{\text {II. } a j 1} \operatorname{lip}(g)\left(\operatorname{lip}(\varphi)+\operatorname{lip}\left(\psi_{\text {II } j} ; \varepsilon\left(\omega_{\text {II. } a j 1} \operatorname{lip}(g)\right)^{-1}\right)\right), \omega_{\text {III } a j 2}, \omega_{\text {II. } a j 3}\right\},
$$

where $\operatorname{lip}(g)$, $\operatorname{lip}(\varphi)$, and $\operatorname{lip}\left(\psi_{\mathrm{II} j} ; \varepsilon\left(\omega_{\mathrm{II} . a j 1} \operatorname{lip}(g)\right)^{-1}\right)$ determined by (19), (20), and (23), respectively.

Proposition 3. For each $j \in\{1,2\}$, it is true that $F_{\text {II. } b j}(N) \in \varepsilon-\operatorname{Lip}\left(W_{\mathrm{II}} ; \mathbb{R}\right)$ and

$$
\begin{aligned}
\operatorname{lip}\left(F_{\text {II. } b j} ; \varepsilon\right) \leqslant \max \{ & \omega_{\text {II. } b j 1} \operatorname{lip}(g)\left(\operatorname{lip}(\varphi)+\operatorname{lip}\left(\psi_{\text {II } j} ; \varepsilon\left(\omega_{\text {II. } b j 1} l i p(g)\right)^{-1}\right)\right), \omega_{\text {II. } b j 2,}, \\
& \left.\omega_{\text {II. } b j 3} \operatorname{lip}(H), \omega_{\text {II. } b j 4} \operatorname{lip}(H), \omega_{\text {II. } b j 5} \operatorname{lip}(H)\left(\rho_{\text {II }} l i p(H) /(8 \varepsilon)+1\right)\right\},
\end{aligned}
$$

where $\operatorname{lip}(g)$, $\operatorname{lip}(\varphi)$, and $\operatorname{lip}\left(\psi_{\mathrm{II} j} ; \varepsilon\left(\omega_{\mathrm{II} . b j 1} \operatorname{lip}(g)\right)^{-1}\right)$ determined by (19), (20), and (23), respectively.

According to our assumptions, we have $M \in W_{i}, i \in\{\mathrm{I}, \mathrm{II}\}$, whence $F_{i j}(M) \geqslant 0$, $j \in\{1,2\}$. These inequalities and Propositions 1-3 imply that problem (16) of finding a projection can be solved by using the algorithms proposed in [2, 3]. Each algorithm either generates an infinite or a finite sequence $Q^{m}$. In the first case, the sequence $Q^{m}$ converges to a zero of function $F_{i j}$. This zero is closest to $M$ under the norm, and the sequence also satisfies inequality $F_{i j}\left(Q^{m}\right)>0$ for each $m=1,2, \ldots$ In the second case, the last element of the sequence is the required point $N_{*}$, in which $F_{i j}\left(N_{*}\right)=0$. In this paper we use one of these algorithms. Before describing it, as we said above, we turn to the weighting coefficients $\omega_{i j l}$ used in constructing the functions $F_{i j}$, because choosing $\omega_{i j l}$ affects the accuracy of the solution obtained by the algorithm.

Since the algorithm finds the next approximation to the optimal solution by using information about possible change in the value of the function $F_{i j}$ with respect to the current approximation and since the function $F_{i j}$ is defined by maximum of some functioncomponents, we must take into account how these function-components are related to each other. If these relations are ignored, then the approximations generated by the algorithm may not reach the stopping criterion $F_{i j}\left(Q^{m}\right)<\varepsilon^{*}$ within a reasonable time (see also remarks 1-2 below). There is a reason for it: the accuracy for a function-component of $F_{i j}$ may be too high, whence the number of algorithm iterations to reach accuracy is too large. It is known (see [5]) that an upper bound of the number of iterations for the gradient-free algorithms, including our algorithm, is determined by using a possible change in the value of the objective function; therefore, if we know these changes for all function-components 
of $F_{i j}$, then we can take $\omega_{i j l}$ to provide the number of iterations adequate to the overall accuracy $\varepsilon^{*}$. In other words, having taken a value of the overall accuracy $\varepsilon^{*}$ for the values of $F_{i j}$, we can specify accuracies for every function-component of $F_{i j}$ by using the values of the corresponding weighting coefficients: accuracy for $l$-th function-component will be equal to $\varepsilon^{*} / \omega_{i j l}$.

In this article, we suppose $\omega_{i j 1}=1, i \in\{$ I, II.a,II.b $\}, j \in\{1,2\}$, that is, the overall accuracy $\varepsilon^{*}$ corresponds to the accuracy of the first function-component of $F_{i j}$. The accuracies $\omega_{i j l}$ for other function-components are taken according to remark above.

Let us present the algorithm.

Step 0. Choose: an initial value $\varepsilon_{0}>0$ of the $\varepsilon$-Lipschitz parameter, the initial projectile velocity magnitude determined by $v$ or $v_{0}$, a lower bound $\kappa$ of $x$, an initial point $N^{0}=M \in W_{i j}$, parameters $\gamma, \lambda \in(0,1)$. Assign the visibility zone constraints determined by $g(\varphi, \psi)$ satisfying (6). Set $Q^{0}:=N^{0}, k:=0$, and $m:=0$.

Step 1. Depending on Problem I, II.a, or II.b, calculate $F_{i j}\left(N^{k}\right), i \in\{$ I, II.a, II.b $\}$, $j \in\{1,2\}$ using (12), (13), or (15), respectively. If $F_{i j}\left(N^{k}\right)<\varepsilon_{k}(1+\gamma)$, then sequentially set $N^{k+1}:=N^{k}, Q^{m+1}:=N^{k}$, and $m:=m+1$ and pass to Step 2. Otherwise, go to Step 3.

Step 2. If $F_{i j}\left(N^{k}\right) \leqslant \varepsilon_{k}$, then set $\varepsilon_{k+1}:=\lambda F_{i j}\left(N^{k}\right)$. Otherwise, set $\varepsilon_{k+1}:=\lambda \varepsilon_{k}$. In either case, go to Step 4.

Step 3. Find $N^{k+1}$ by the following scheme. Depending on Problem I, II.a, or II.b, calculate $\operatorname{lip}\left(F_{i j}, \varepsilon_{k}\right), i \in\{$ I, II.a,II.b $\}, j \in\{1,2\}$ by formulas (22), (24), or (25), respectively, using (19)-(21), and (23). Solve the problem

$$
N^{k+1}=\arg \min \left\{F_{i j}(X) \mid X \in \text { fr } K_{k} \cap W_{i j}\right\}, i \in\{\text { I, II.a, II.b }\}, j \in\{1,2\},
$$

where

$$
K_{k}=\left\{X \in \mathbb{R}^{n} \mid\|X-M\| \leqslant \sum_{s=0}^{k} r_{s}\right\}, r_{k}=\frac{F_{i j}\left(N^{k}\right)-\varepsilon_{k}}{\sqrt{n} \operatorname{lip}\left(F_{i j} ; \varepsilon_{k}\right)},
$$

$n=2$ for Problem I and $n=3$ for Problems II.a and II.b. Next, set $\varepsilon_{k+1}:=\varepsilon_{k}$ and go to Step 4.

Step 4. If $F_{i j}\left(N^{k+1}\right)=0$ or $F_{i j}\left(Q^{m}\right)=0$, then $N^{k+1}$ or $Q^{m}$ is regarded as a solution of problem (16), respectively. Otherwise, set $k:=k+1$ and pass to Step 1.

Remark 1. According to the main statement about the algorithm convergence (see [2]), if the number of points $N^{k}$ is infinity, then the algorithm ensures the convergence $F_{i j}\left(Q^{m}\right) \underset{m \rightarrow \infty}{\longrightarrow} 0$. Therefore, the condition of the form $F_{i j}\left(Q^{m}\right)<\varepsilon^{*}, \varepsilon^{*} \in(0,1)$, should be included in the stopping criterion. We can also include the conditions setting accuracies for some function-components of $F_{i j}$ or the condition of the form $\left\|Q^{m}-Q^{m+1}\right\|<\varepsilon_{Q}^{*}$, $\varepsilon_{Q}^{*} \in(0,1)$ in the stopping criterion.

Remark 2. It is clear that the main computational costs are produced by Step 3, where the optimization subproblem for finding the point $N^{k+1}$ is solved. We will give two pieces of advice about solving this subproblem. First, since each function $F_{i j}, j \in\{1,2\}$, is minimized either on a circle (Problem $i=\mathrm{I}$ ) or on a 2 -sphere (Problems $i=$ II.a, II.b), it follows that the dimension of the subproblem can be reduced by one by applying transformation to polar coordinates or spherical ones, respectively. Secondly, it is not necessary to solve this subproblem precisely. Indeed, since the value $F_{i j}\left(N^{k}\right)$ is compared 
with $\varepsilon_{k}$ and $\varepsilon_{k}(1+\gamma)$, instead of the point $N^{k+1}$ determined by (26), one can find a point $\tilde{N}^{k+1}$ such that $F_{i j}\left(N^{k+1}\right) \leqslant F_{i j}\left(\tilde{N}^{k+1}\right)<F_{i j}\left(N^{k+1}\right)+\delta_{k}$, where $\delta_{k}<\varepsilon_{k} \gamma$.

Remark 3. For computing values of the 4-th function-component of $F_{\text {II.b }}$ in problem II.b, we must solve the one-dimensional optimization problem of minimization of the function $f(\lambda)=H(\lambda N+(1-\lambda) M)$. This problem can be solved by some corresponding numerical method. A similar remark also concerns the 5 -th function-component of $F_{\text {II.b }}$.

\section{Numerical Examples}

The algorithm was tested on several examples in which the target $M$, the set $W_{i}$, $i \in\{\mathrm{I}, \mathrm{II}\}$, and the overall accuracy $\varepsilon^{*}$ that taken equal to an initial value $\varepsilon_{0}$ of the $\varepsilon$ Lipschitz parameter were varied. The optimization subproblem for finding the point $N^{k+1}$ (see Step 3) was solved by the uniform search method.

For all examples, the following parameter values were set: $v_{0}=180 \mathrm{~m} / \mathrm{s} ; \kappa=100 \mathrm{~m}$; $\gamma=\lambda=0,5$. The values of the variable parameters are presented in Tables $1-3$. Hereinafter and in Tables $1-3$, we use the following notation: $M_{1}=(110,0,20), M_{2}=(2700,0,-10)$ are the target $M$ (the third coordinate of $M$ for Problem I was equal to 0$) ; E_{1}=\{(\varphi, \psi) \mid$ $\varphi \in[0,2 \pi], \psi \in[7 \pi / 36,8 \pi / 36]\}, E_{2}=\{(\varphi, \psi)|\varphi \in[0,2 \pi]| 4+,\sin \varphi \mid \pi / 36 \leqslant \psi \leqslant$ $|1+\sin \varphi| \pi / 9\} ;\left(x_{N}, y_{N}, z_{N}\right)$ is the solution of Problem I, II.a, or II.b; $(\varphi, \psi)$ is the angles for the direction of the gun barrel, which ones corresponding to $\left(x_{N}, y_{N}, z_{N}\right) ; k_{\text {tot }}$ is the total number of algorithm iterations; $t$ is the total computational time in seconds. The value $z_{\min }$ in Problems II.a and II.b was equal to -10 , and the set $D$ in Problems II.b was determined by function

$$
H(x, y, z)=\min \{\max \{90-x, x-130,-10-y, y-30, z-20\}, z+10\} .
$$

Table 1

Results of test examples for Problem I

\begin{tabular}{|l|l|l||l|l|l|l|l|l|}
\hline$M$ & $E$ & $\varepsilon$ & $x_{N}, \mathrm{~m}$ & $y_{N}, \mathrm{~m}$ & $\varphi^{\circ}$ & $\psi,^{\circ}$ & $k_{\text {tot }}$ & $t, \mathrm{~s}$ \\
\hline$M_{1}$ & \multirow{2}{*}{$E_{1}$} & 0,1 & 2818,6 & 0,0 & 0,0 & 29,3 & 300 & 0,146 \\
\cline { 3 - 9 } & & 0,05 & 2976,8 & 0,0 & 0,0 & 32,1 & 353 & 0,170 \\
\cline { 3 - 9 } & 0,01 & 3081,4 & 0,0 & 0,0 & 34,4 & 575 & 0,306 \\
\cline { 3 - 8 } & \multirow{2}{*}{$E_{2}$} & 0,1 & 1523,4 & $-152,1$ & $-5,7$ & 13,8 & 301 & 0,419 \\
\cline { 3 - 8 } & & 0,05 & 1834,6 & $-91,4$ & $-2,9$ & 16,9 & 442 & 0,822 \\
\cline { 3 - 8 }$M_{2}$ & \multirow{2}{*}{$E_{1}$} & 0,01 & 2068,1 & $-20,6$ & $-0,6$ & 19,4 & 891 & 3,514 \\
\cline { 3 - 8 } & & 0,05 & 2820,3 & 0,0 & 0,0 & 29,3 & 40 & 0,034 \\
\cline { 3 - 8 } & & 0,01 & 3081,4 & 0,0 & 0,0 & 34,4 & 331 & 0,213 \\
\cline { 3 - 8 } & \multirow{2}{*}{$E_{2}$} & 0,1 & 2606,4 & 48,4 & 1,1 & 26,0 & 36 & 0,031 \\
\cline { 3 - 8 } & 0,05 & 2447,8 & 138,6 & 3,2 & 24,0 & 158 & 0,086 \\
\cline { 3 - 8 } & 0,01 & 2316,7 & 217,0 & 5,4 & 22,4 & 456 & 0,244 \\
\hline
\end{tabular}

The weighting coefficients in the functions $F_{\text {I }}, F_{\text {II.a } j}$, and $F_{\text {II.b } j}$ (see (12), (13), and (15)) were chosen as follows: I) $\omega_{\text {I } j 1}=1, \omega_{\text {I } j 2}=0,01$; II.a) $\omega_{\text {II.a } j 1}=1, \omega_{\text {II.a } j 2}=\omega_{\text {II.a } j 3}=0,01$; II.b) $\omega_{\text {II.b } j 1}=1, \omega_{\text {II.b } j 2}=0,01, \omega_{\text {II.b } j 3}=\omega_{\text {II.b } j 4}=\omega_{\text {II.b } j 5}=0,001$. 
Results of test examples for Problem II.a

\begin{tabular}{|l|l|l||l|l|l|l|l|l|l|}
\hline$M$ & $E$ & $\varepsilon$ & $x_{N}, \mathrm{~m}$ & $y_{N}, \mathrm{~m}$ & $z_{N}, \mathrm{~m}$ & $\varphi^{\circ}$ & $\psi^{\circ}$ & $k_{\text {tot }}$ & $t, \mathrm{~s}$ \\
\hline$M_{1}$ & \multirow{2}{*}{$E_{1}$} & 0,1 & 100,0 & 0,1 & 58,0 & 0,1 & 31,0 & 1889 & 1,249 \\
\cline { 3 - 9 } & & 0,05 & 100,0 & 1,8 & 65,4 & 1,0 & 34,1 & 4182 & 2,244 \\
\cline { 3 - 9 } & & 0,01 & 102,0 & 2,1 & 72,1 & 1,2 & 36,1 & 26297 & 5,227 \\
\cline { 2 - 9 } & \multirow{2}{*}{$E_{2}$} & 0,1 & 108,7 & $-0,5$ & 25,9 & $-0,3$ & 14,3 & 614 & 1,073 \\
\cline { 3 - 9 } & & 0,05 & 106,8 & $-1,0$ & 31,4 & $-0,6$ & 17,3 & 2444 & 1,495 \\
\cline { 3 - 9 } & & 0,01 & 104,8 & $-0,3$ & 35,9 & $-0,2$ & 19,8 & 17619 & 8,854 \\
\hline \multirow{3}{*}{$E_{2}$} & 0,1 & 2730,7 & 0,0 & 48,3 & 0,0 & 29,3 & 8478 & 4,064 \\
\cline { 3 - 9 } & & 0,05 & 2771,2 & 0,0 & 121,4 & 0,0 & 32,2 & 33069 & 12,750 \\
\cline { 3 - 9 } & & 0,01 & 2803,2 & $-0,1$ & 173,5 & 0,0 & 34,4 & 213343 & 103,261 \\
\cline { 3 - 9 } & \multirow{2}{*}{$E_{2}$} & 0,1 & 2502,2 & 107,1 & $-8,6$ & 2,5 & 24,4 & 27281 & 5,952 \\
\cline { 3 - 9 } & & 0,05 & 2336,3 & 826,1 & $-4,3$ & 19,5 & 24,2 & 244288 & 131,227 \\
\cline { 3 - 9 } & & 0,01 & 2324,5 & 663,2 & $-2,8$ & 15,9 & 23,4 & 313626 & 163,866 \\
\hline
\end{tabular}

Table 3

Results of test examples for Problem II.b

\begin{tabular}{|l|l|l||l|l|l|l|l|l|l|}
\hline$M$ & $E$ & $\varepsilon$ & $x_{N}, \mathrm{~m}$ & $y_{N}, \mathrm{~m}$ & $z_{N}, \mathrm{~m}$ & $\varphi^{\circ}$ & $\psi,^{\circ}$ & $k_{\text {tot }}$ & $t, \mathrm{~s}$ \\
\hline$M_{1}$ & $E_{1}$ & 0,1 & 100,0 & 0,0 & 58,0 & 0,0 & 31,0 & 2748 & 9,753 \\
\cline { 3 - 10 } & & 0,05 & 100,2 & 16 & 62,6 & 9,1 & 32,6 & 7308 & 14,240 \\
\cline { 2 - 9 } & \multirow{2}{*}{$E_{2}$} & 0,1 & 108,7 & $-0,5$ & 25,9 & $-0,3$ & 14,3 & 1072 & 11,303 \\
\cline { 3 - 9 } & & 0,05 & 106,8 & $-1,0$ & 31,4 & $-0,6$ & 17,3 & 4258 & 11,179 \\
\hline \multirow{2}{*}{$M_{2}$} & \multirow{2}{*}{$E_{1}$} & 0,1 & 2730,7 & 0,0 & 48,3 & 0,0 & 29,3 & 12746 & 19,626 \\
\cline { 3 - 9 } & & 0,05 & - & - & - & - & - & - & - \\
\cline { 2 - 9 } & \multirow{2}{*}{$E_{2}$} & 0,1 & 2502,2 & 107,1 & $-8,6$ & 2,5 & 24,4 & 47995 & 88,068 \\
\cline { 3 - 9 } & & 0,05 & 2437,0 & 145,7 & $-10,0$ & 3,4 & 23,6 & 69230 & 121,723 \\
\hline
\end{tabular}

The tests were performed on an Intel Core i3-4020 1,50 GHz personal computer. The numerical results are presented in Tables 1-3. It follows that the algorithm finds the $\varepsilon$ solution in Problem I faster than in other two Problems. Moreover, in Problem II.a and especially in Problem II.b, there is a large increase in the number of iterations with $\varepsilon_{0}$ greater than 0,05 . (The dash in Table 3 means that the algorithm did not give a solution for $200 \mathrm{~s}$.) A reasonable explanation for this increase may be that, for the functions $F_{\text {II.a,b }}$ a decrease in $\varepsilon$ implies an increase in $L(\varepsilon)$. However, for a finite number, number of iterations (when $L(\varepsilon)$ is not very high), it is still possible to cut off a set that does not contain zero of $F_{i j}$; this yields that the set $W_{i}$ is narrowed down to its subset. It is readily seen that computational time can be reduced by parallelizing some parts of the algorithm. For example, one can be applied in subproblems, in which the uniform search method are used. 


\section{References}

1. Konovalov A.A. Vneshnyaya ballistika [External Ballistics]. Moscow, Tsentral'nyy nauchnoissledovatel'skiy institut informacii, 1979. (in Russian)

2. Arutyunova N.K., Dulliev A.M., Zabotin V.I. Algorithms for Projecting a Point onto a Level Surface of a Continuous Function on a Compact Set. Computational Mathematics and Mathematical Physics, 2014, vol. 54, no. 9, pp. 1395-1401. DOI: 10.7868/S0044466914090038

3. Zabotin V.I., Arutyunova N.K. [Two Algorithms for Finding the Projection of a Point onto a Nonconvex Set in a Normed Space]. Computational Mathematics and Mathematical Physics, 2013, vol. 53, no. 3, pp. 344-349. DOI: 10.7868/S0044466913030162 (in Russian)

4. Vanderbei R.J. Extension of Piyavskii's Algorithm to Continuous Global Optimization. Journal of Global Optimization, 1999, vol. 14, pp. 205-216.

5. Nesterov Yu. Vvedenie v vypukluyu optimizaciyu [Introduction into a Convex Optimization]. Moscow, Moskovskiy tsentr nepreryvnogo matematicheskogo obrazovaniya, 2010. (in Russian)

6. Arutyunova N., Dulliev A., Zabotin V. Models and Methods for Three External Ballistics Inverse Problems. Available at: http://arxiv.org/abs/1610.02933 (accessed 16 October 2017).

Received January 20, 2017

УДК $519.863+519.853 .4+519.853 .6+531.554$

DOI: $10.14529 / \operatorname{mmp} 170408$

МОДЕЛИ И МЕТОДЫ ДЛЯ ТРЕХ ОБРАТНЫХ ЗАДАЧ ВНЕШНЕЙ БАЛЛИСТИКИ

\section{Н.К. Арутюнова, А.М. Дуллиев, В.И. Заботин}

Казанский национальный исследовательский технический университет им. А.Н. Туполева - КАИ, г. Казань, Российская Федерация

Рассматриваются три математические модели задачи выбора оптимального направления ствола орудия при стрельбе неуправляемым снарядом в предположении, что полуось ствола может перемещаться в связном невыпуклом конусе, имеющем негладкую боковую поверхность и моделирующем ограничения на зону видимости. В первой задаче цель расположена в плоскости истинного горизонта орудия, во второй и третьей - в некоторой области пространства. Отличительной особенностью моделей является $\varepsilon$-липшицевость целевых функций. Построен единый численный метод решения поставленных задач, базирующийся на одном алгоритме проектирования точки на множество уровня $\varepsilon$-липшицевой функции. На его основе составлена программа для ЭВМ. По каждой из задач проведена серия вычислительных экспериментов.

Ключевые слова: математическое моделирование; обратнал задача внешней баллистики; оптимизация; проекция на невыпуклое множество; в-липшицевость; приближенное решение.

\section{Литература}

1. Коновалов, А.А. Внешняя баллистика / А.А. Коновалов. - М.: Центральный научноисследовательский институт информации, 1979.

2. Арутюнова, Н.К. Алгоритмы проектирования точки на поверхность уровня непрерывной на компакте функции / H.K. Арутюнова, А.М. Дуллиев, В.И. Заботин // Журнал вычислительной математики и математической физики. - 2014. - Т. 54, № 9. C. $1448-1454$. 
3. Заботин, В.И. Два алгоритма отыскания проекции точки на невыпуклое множество в нормированном пространстве / В.И. Заботин, Н.К. Арутюнова // Журнал вычислительной математики и математической физики. - 2013. - Т. 53, № 3. - С. 344-349.

4. Vanderbei, R.J. Extension of Piyavskii's Algorithm to Continuous Global Optimization / R.J. Vanderbei // Journal of Global Optimization. - 1999. - V. 14. - P. 205-216.

5. Нестеров, Ю.Е. Введение в выпуклую оптимизацию / Ю.Е. Нестеров. - М.: МЦНМО, 2010.

6. Arutyunova, N. Models and Methods for Three External Ballistics Inverse Problems / N. Arutyunova, A. Dulliev, V. Zabotin. - URL: https://arxiv.org/abs/1610.02933.

Наталья Константиновна Арутюнова, кандидат физико-математических наук, доцент, кафедра «Прикладная математика и информатика», Казанский национальный исследовательский технический университет им. А.Н. Туполева - КАИ (г. Казань, Российская Федерация), NKArutyunova@kai.ru.

Айдар Мансурович Дуллиев, кандидат физико-математических наук, доцент, кафедра «Прикладная математика и информатика», Казанский национальный исследовательский технический университет им. А.Н. Туполева - КАИ (г. Казань, Российская Федерация), AMDulliev@kai.ru.

Владислав Иванович Заботин, доктор технических наук, профессор, кафедра «Прикладная математика и информатика», Казанский национальный исследовательский технический университет им. А.Н. Туполева - КАИ (г. Казань, Российская Федерация), VIZabotin@kai.ru.

Поступила в редакцию 20 января 2017 г. 